\title{
SIMULAÇÃO E CONTROLE DE UM REATOR DE POLIMERIZAÇÃO DE PROPENO COM O SOFTWARE EMSO
}

\author{
A. C. S. R. DIAS ${ }^{1}$, W. B. da SILVA ${ }^{2}$ e J. C. S. DUTRA ${ }^{3}$ \\ LAMCES - Laboratório de Métodos Computacionais, Controle e Estimação \\ Universidade Federal do Espírito Santo - Engenharia Química (campus de Alegre) \\ ${ }^{1}$ ana_csrd@hotmail.com; ${ }^{2}$ wellingtonuff@yahoo.com.br; ${ }^{3}$ juliosdutra@yahoo.com.br \\ RESUMO - O polipropileno tem grande importância econômica, o que estimula \\ buscas por melhoria em seu processo produtivo. Para tal, a simulação de \\ processos, testando modelos matemáticos, tem sido muito utilizada por ser um \\ procedimento simples e de baixo custo. Neste trabalho, utilizando-se o software \\ EMSO, estudos computacionais foram realizados, envolvendo modelagem, \\ simulação e controle de um reator de polimerização de propeno. Algumas \\ adaptações de código computacional foram necessárias para a implementação do \\ modelo. Usando o pacote DASSLC, a simulação foi rápida e robusta, levando a \\ resultados consistentes que podem ser utilizados para o monitoramento e controle \\ da polimerização. Dessa forma, mostrou-se que o EMSO é uma ferramenta \\ eficiente e de grande potencial.
}

\section{INTRODUÇÃO}

O polipropileno (PP) é uma resina que apresenta alta resistência a impactos e a fratura, fácil moldagem, baixo custo e muito utilizada em diversos setores industriais (Dutra et al., 2014; Chum e Swogger, 2008). Existem diversos processos de produção de PP, combinando tecnologias de produção e de catalisadores, realizados por meio de solução, suspensão em um solvente (slurry), em massa (bulk) ou em fase gasosa. Um processo bastante comum baseia-se na tecnologia LIPP (LIquid Pool Polymerization), que utiliza a polimerização em suspensão de propeno líquido em um reator mecanicamente agitado operando em alta pressão (Dutra et al., 2014; Ferreira e Faezipour, 2012).

Para se compreender os problemas operacionais do processo produtivo ou o efeito de diversas variáveis, de forma antecipada e preditiva, recomenda-se o uso de simuladores. Essas ferramentas são extremamente importantes porque permitem, por exemplo, encontrar condições ideais de operação, avaliar mudanças em instalações industriais sem afetar a operação real e reduzir o tempo de decisão em relação às políticas operacionais (Soares e Secchi, 2003; Ferreira e Faezipour, 2012).

Existem diversos simuladores computacionais que podem ser empregados para estes fins. Mas, cabe destaque àqueles que conferem flexibilidade para lidar com diversas situações e que sejam de código livre, como o software EMSO. Especificamente, esta ferramenta consiste de um ambiente computacional voltado para a modelagem, otimização de processos, projeto de estruturas de controle, estimação de parâmetros e reconciliação de dados (Soares e Secchi, 2003). 
Neste contexto, o presente trabalho aborda a questão da simulação e do controle do processo de polimerização de propeno com o software EMSO, empregando a modelagem proposta por Dutra et al. (2014). Durante a implementação do novo algoritmo, mudanças na representação do modelo do processo foram feitas para adequar à linguagem computacional do EMSO. Os resultados mostram um bom desempenho do simulador escolhido, que operou com rapidez e robustez.

\section{O SOFTWARE EMSO}

A simulação de um processo permite prever o comportamento do sistema, com um dado grau de confiança, baseando-se em informações de entrada e parâmetros, de acordo com o conjunto de considerações especificado. Os simuladores são ferramentas importantes no projeto e no desenvolvimento de processos industriais, pois possibilitam, por exemplo, a validação de projetos ou modificações em uma planta industrial sem influenciar sua operação real. Permitem avaliar sua operabilidade prática, como também possíveis aumentos de produção e reduções de custo. As alterações são feitas no simulador, seus resultados são analisados e, só depois, se confirmadas mais eficientes, são aplicadas no processo real. Com isso, é possível reduzir o tempo envolvido no processo decisório e na aplicação de novas condições operacionais (Quinto et al., 2009).

A simulação de processos é uma prática crescente em ambientes de produção. Entretanto, as ferramentas disponíveis no mercado são consideradas insuficientes pelos usuários, principalmente pela flexibilidade limitada dos softwares, dificuldade de utilização e aprendizagem, e pelo alto custo de aquisição. Com o intuito de proporcionar maior flexibilidade aos usuários, o projeto Ambiente Livre para Simulação, Otimização e Controle de Processos (ALSOC) da Universidade Federal do Rio Grande do Sul, em parceria com outras universidades e petroquímicas nacionais, desenvolveu o EMSO. Aspectos positivos dos simuladores mais utilizados foram mantidos e novos métodos foram desenvolvidos para suprir características ausentes. O paradigma de programação orientada a objetos foi utilizado, permitindo trabalhar com conceitos de objeto, herança e composição, possibilitando melhor organização e versatilidade (Quinto et al., 2009; Soares e Secchi, 2003).

O EMSO é um ambiente gráfico integrado para o desenvolvimento de modelos e não apresenta custo para utilização em instituições de ensino. Trata-se de um simulador baseado em equacionamento, o que o torna flexível para lidar com diversas situações. A modelagem é realizada por uma linguagem de alto nível, bem estruturada e de fácil aprendizagem, o que o torna um software apropriado para muitas aplicações em pesquisa e ensino (Soares e Secchi, 2003). Portanto, os esforços neste trabalho convergiram para o desenvolvimento de um algoritmo com linguagem de modelagem adequada à plataforma do EMSO.

\section{POLIMERIZAÇÃO DE PROPENO}

Diversos processos de polimerização podem ser utilizados para a produção de polipropileno, combinando tecnologias de produção e de catalisadores. A escolha do catalisador depende das propriedades do PP que se deseja obter (Dutra et al., 2014).

Para atender as exigências do mercado, os processos de polimerização devem ser projetados para operar em diversas formas e produzir resinas com diferentes propriedades. 
Duas soluções possíveis para esta situação podem ser assim descritas: a) utilizar diferentes reatores para as diferentes condições de operação, o que gera custos elevados e exige grande espaço físico; ou, b) diferentes sistemas catalíticos. Esta segunda opção requer estudos sobre os possíveis problemas de controle que surgem, pois os catalisadores apresentam cinéticas diferentes que afetam o comportamento do processo e mudam para cada sistema selecionado (Dutra et al., 2014).

Para o desenvolvimento das estratégias de controle, é necessário um modelo matemático que permite a análise do comportamento e a determinação das condições operacionais. Na literatura, existem várias contribuições, contudo, uma referência recente é o estudo de Dutra et al (2014), que considera a produção de PP em fase líquida - a tecnologia LIPP.

\subsection{Modelagem do Processo}

O modelo proposto por Dutra et al. (2014) utiliza um reator contínuo de tanque agitado (CSTR), operando em alta pressão, utilizando três tipos diferentes de catalisadores. Propileno líquido é usado como meio de suspensão para as partículas de polímero; trietil-alumínio (TEA) e para-etox-etil-benzoato (PEEB) são utilizados como aditivos. O hidrogénio participa como um agente de transferência de cadeia.

Por simplicidade, neste presente trabalho, o sistema de três catalisadores foi substituído por um único tipo de catalisador, no caso Ziegler-Natta, para a produção de polímero com elevada massa molar e baixa rigidez. Selecionou-se esse sistema por atender a maioria das aplicações industriais e por apresentar a reação de transferência de cadeia com hidrogênio, o que é muito importante no controle do tamanho da cadeia polimérica.

\subsection{Configuração de Controle}

O sistema de controle proposto por Dutra et al. (2014) é dividido em duas camadas hierárquicas. A camada inferior (controle regulatório) garante a estabilidade do processo. Ela contempla as malhas de controle do volume do reator $(\mathrm{V})$, das temperaturas do reator $(\mathrm{T})$, da saída de líquido refrigerante (Tw) e da produtividade. A camada superior (controle supervisório) atenua os efeitos das perturbações sobre a estabilidade do processo. É composta pelas malhas de controle do teor de solúveis em xileno (XS) e do índice de fluidez (MI). Os controladores PI foram implementados para ambas as camadas de acordo com o algoritmo de velocidade discreta dado pela Equação 1.

$$
u\left(t_{k}\right)=u\left(t_{k-1}\right)+k c\left(e\left(t_{k}\right)-e\left(t_{k-1}\right)+T s \frac{e(t k)}{\tau_{I}}\right)
$$

Nesta representação $u\left(t_{k}\right)$ é a variável controlada no instante $\mathrm{k} \mathrm{e}^{u\left(t_{k-1}\right)}$, no instante $\mathrm{k}$ 1. $k c$ é o ganho do controlador, ${ }^{e\left(t_{k}\right)} \mathrm{e}^{e\left(t_{k-1}\right)}$ são, respectivamente, os erros entre o valor do set point e o valor da variável medida nos instantes $\mathrm{k}$ e k-1, Ts é o tempo de amostragem e $\mathrm{\tau}_{I}$ é a constante de tempo integral do controlador. 


\subsection{Implementação do Modelo}

Durante a implementação das estruturas de controle, deparou-se com um problema de compatibilidade de linguagem computacional. O EMSO não permite a consideração de variáveis contínuas e discretas na mesma equação, embora considere variáveis inteiras em laços do tipo FOR-END. Neste caso, os termos envolvendo a diferença entre um dado do tempo atual e o do tempo anterior (isto é, $t_{k}-t_{k-1}$ ) foram considerados derivadas de primeira ordem em relação ao tempo, permitindo a implementação do controlador na forma de velocidade (Equação 2). O bias do controlador foi tomado como o valor inicial deste, $\mathrm{u}\left(\mathrm{t}_{0}\right)$, e os novos valores, $u(t)$, das variáveis manipuladas foram determinados durante a integração do sistema de equações diferenciais do modelo juntamente com as equações que definem os controladores.

$$
\frac{d u(t)}{d t}=k c\left(\frac{d e(t)}{d t}+T s \frac{e(t k)}{\tau_{I}}\right)
$$

As equações do controlador foram alteradas pela adição de uma estrutura condicional (IF-ELSE-END) para limitar entre valores mínimos e máximos a manipulação das vazões utilizadas pelas camadas de controle, de modo a evitar problemas numéricos.

Para as simulações, utilizou-se um método numérico Dasslc (Secchi, 1992) para solução das equações algébrico-diferenciais.

De acordo com o registro de simulação fornecido pelo EMSO, o índice de estrutura diferencial (structural differential index) deste problema é 1 e há 68 variáveis, 65 equações e 3 especificações, fornecendo grau de liberdade igual a 0 . Dentre as equações, 25 são diferenciais ordinárias.

\section{RESULTADOS}

Para testar o desempenho das estruturas de controle, aplicaram-se degraus de magnitude 10 nos set points das malhas de controle do teor de solúveis em xileno (XS), que tem grande impacto na qualidade final do polímero, e da temperatura do reator (T), que influencia diretamente na atividade catalítica.

As Figuras 1a e 1c mostram, respectivamente, os resultados de simulação para o teor de solúveis em xileno e para a temperatura do reator em seus valores nominais. Por outro lado, as Figuras $1 \mathrm{~b}$ e 1d apresentam o comportamento de XS e de T após a aplicação do degrau em seus respectivos set points. Como se pode observar, as estruturas de controle implementadas mostraram-se satisfatórias, e, quando aplicado o degrau em seus respectivos set points, resultou no aumento do valor das variáveis controladas (teor de extraíveis em xileno e temperatura do reator) atingindo os novos valores de set points (mantendo-os nestas novas condições) com ação rápida e suave (smooth).

Foi aplicado também um degrau de magnitude $10 \mathrm{~K}$ na temperatura de alimentação do monômero (Figura 2), atuando como uma perturbação para a malha de controle da temperatura do reator. Mesmo na presença de perturbação, o controlador foi capaz de retornar o valor da variável controlada (temperatura do reator) para seu set point com robustez. 
Figura 1 - (a) Perfil do teor de solúveis em xileno, (b) Perfil de XS após aplicação do degrau de magnitude $10 \mathrm{em}$ seu set point, (c) Perfil da temperatura do reator, (d) Perfil de T após aplicação do degrau de magnitude $10 \mathrm{em}$ seu set point.

a)

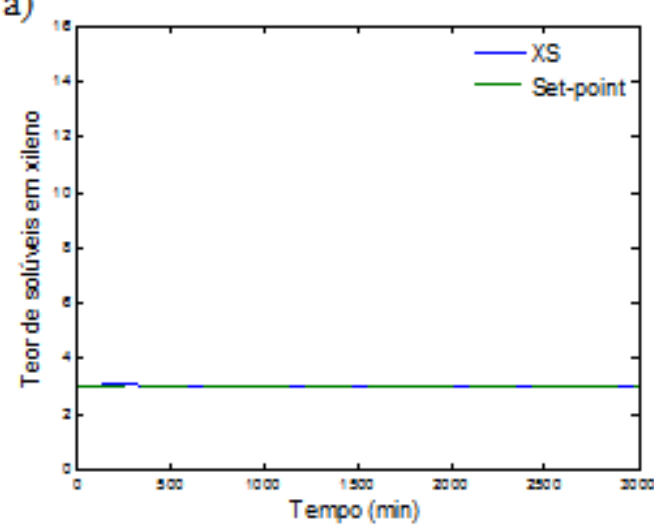

c)

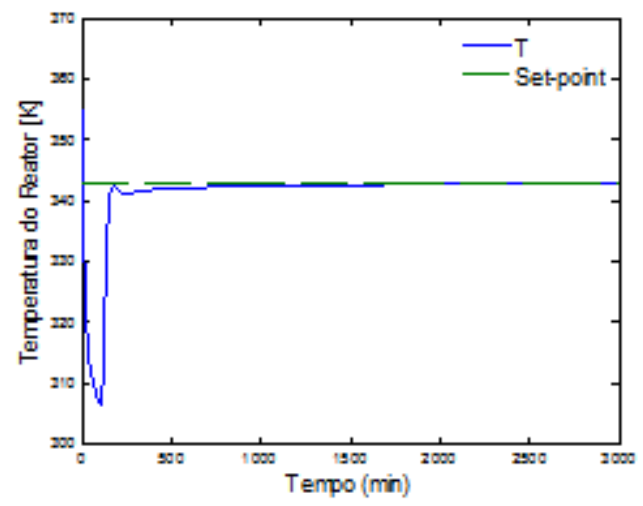

b)

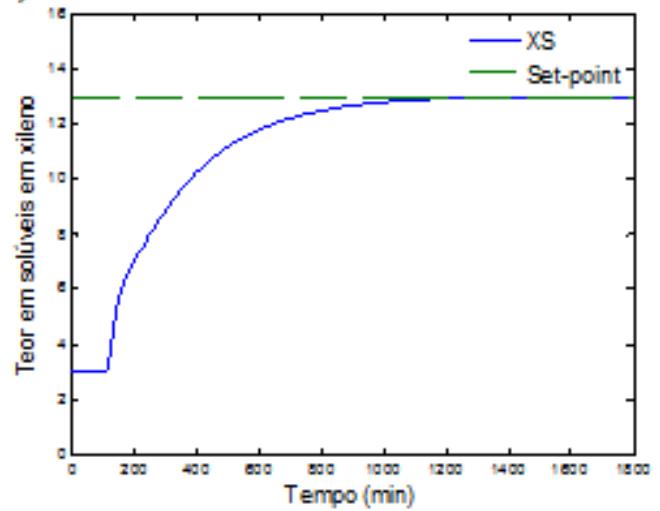

d)

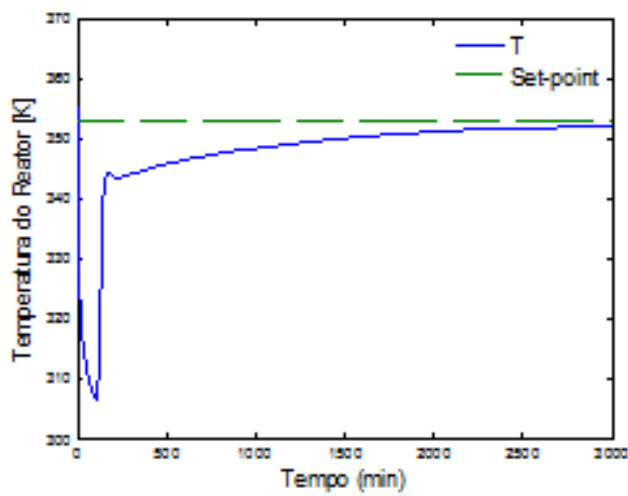

Figura 2 - Perfil da temperatura de saída do reator frente a uma perturbação na temperatura de entrada de monômero no reator.

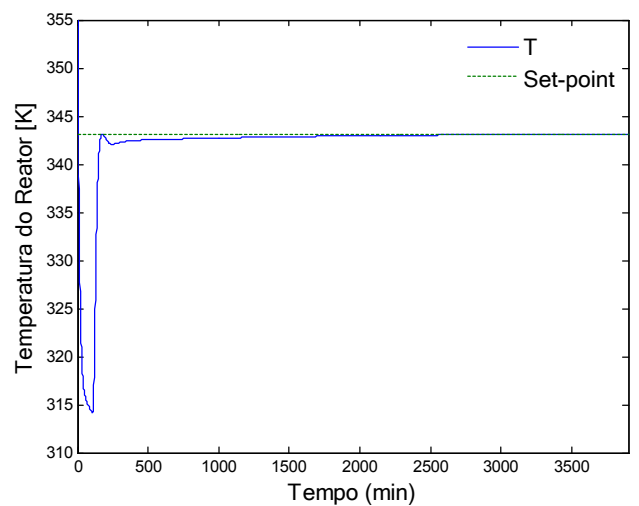




\section{CONCLUSÃO}

Neste trabalho, estudaram-se a modelagem matemática e o problema de controle propostos por Dutra et al. (2014) para o processo de polimerização de propeno em massa, sendo que a contribuição central desta pesquisa foi o emprego do software EMSO para as tarefas de simulação dinâmica e controle.

Esta ferramenta de simulação foi utilizada com sucesso; no entanto, exigiu que fossem feitas adaptações em algumas representações do modelo, como a transformação de variáveis discretas em contínuas. O teste servo realizado para as malhas de controle do teor de solúveis em xileno e da temperatura do reator; e, o teste regulatório para a temperatura do reator; mostraram resultados satisfatórios. Isto confirma a eficácia das estruturas de controle propostas por Dutra et al. (2014) e a potencialidade do EMSO em relação à integração numérica rápida e robusta das equações com o pacote DASSLC.

O modelo implementado em EMSO pode ser usado em trabalhos futuros na aplicação de outras ferramentas de controle, como a estimação de estado para o controle inferencial; na otimização das condições de operação (a fim de reduzir a utilização de um catalisador, por exemplo); e, na implementação de políticas operacionais ótimas de controle preditivo.

\section{REFERÊNCIAS}

CHUM, P. S.; SWOGGER, K. W. Olefin polymer Technologies - History and recent progress at the Dow Chemical Company. Progress in Polymer Science, v. 33, p. 797$819,2008$.

DUTRA, J. C. S.; FEITAL, T. S.; SKOGESTAD, S.; LIMA, E. L.; PINTO, J. C. Control of bulk propylene polymerizations operated with multiple catalysts through controller reconfiguration. Macromolecular Reaction Engineering, v. 8, p. 201-216, 2014.

FERREIRA, S.; FAEZIPOUR, M. An analysis of processes, risks, and best practices for use in developing systems engineering process simulators. Procedia Computer Science, v. 8, p. 87-92, 2012.

QUINTO, T. C.; SECCHI, A. R.; BISCAIA JR., E. C. A continuous implementation of the ideal time delay in EMSO. Computer Aided Chemical Engineering, v. 27, p273-278, 2009.

SECCHI, A. R. DASSLC User's Manual Version 1.0, Universidade Federal do Rio Grande do Sul, DEQUI, Porto Alegre, RS, Brasil, 1992

SOARES, R. P.; SECCHI, A. R. EMSO: A new environment for modelling, simulation and optimisation. Computer-Aided Chemical Engineering, v. 14, p. 947-952, 2003. 